\title{
Marine vibrios associated with superficial septic lesions
}

\author{
W. J. RYAN \\ From the Public Health Laboratory, Exeter EX2 5AD
}

SYNOPSIS Three cases are reported in which a marine vibrio, Vibrio alginolyticus, was isolated from superficial septic lesions. All cases had been exposed to sea-water. The possible significance of these findings and the need for further investigations are discussed.

Although the marine vibrio, Vibrio parahaemolyticus, is now well established as a cause of human gastroenteritis, the association of other marine vibrios with disease in man has received little attention. The following is a report of the isolation of Vibrio alginolyticus, a sea-water vibrio closely related to $V$. parahaemolyticus, from superficial septic lesions in three patients exposed to a marine environment but with no other known connection between them. The infections occurred in three different towns along the south Devon-Dorset coast.

\section{Case reports}

CASE 1

A man aged 43 years developed a septic thumb which failed to respond to ampicillin therapy. The lesion was incised and subsequently resolved without further antibiotic treatment. A profuse pure growth of a Gram-negative bacillus, which was later identified as $V$. alginolyticus, was obtained from the pus. Enquiry revealed that the patient was a fisherman and that the lesion had developed after injury to the thumb from either a fish bone or a piece of shell.

CASE 2

A boy aged 11 years had a superficial septic leg wound. Pus from the lesion gave a moderate pure growth of a Gram-negative rod also later shown to be $V$. alginolyticus. On enquiry it was found that the lesion was the result of an injury received when clambering on rocks on the sea shore. The further outcome of this infection is not known as the patient was a visitor who left the district.

Received for publication 31 March 1976

\section{CASE 3}

A man aged 32 years had an acute otitis externa. A 음 swab gave a profuse pure growth of an organism which proved to be $V$. alginolyticus. It was later $Z$ found that, although the patient had had previous attacks of this complaint, he attributed his present condition to bathing in the sea. The lesion responded to conventional treatment.

\section{Methods}

Except where indicated, materials and techniques were as described by Cowan and Steel (Cowan 1974). Pus was plated on horse blood agar plates for aerobic and anaerobic culture and on CLED (cystinelactose-electrolyte-deficient) agar (Mackey and Sandys, 1966). A McIntosh and Fildes jar with a cold catalyst was used for anaerobic culture. Primary cultures were incubated at $37^{\circ} \mathrm{C}$.

\section{Results}

After overnight incubation growth was readily apparent on both aerobic and anaerobic blood agar plates but there was no growth on CLED agar. Aerobic growth was slightly better than anaerobic. 음 Colonies at this stage were about 1 to $2 \mathrm{~mm}$ in $D$ diameter, smooth, round, convex, greyish, translucent, and rather like small coliform colonies; later $N$ they became larger, more irregular, and rather tenacious. Stained films showed a highly pleomorphic

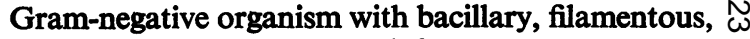
coccoid, and swollen round forms. Some commashaped cells were usually seen. The cells were highly 0 motile. No capsules or spores were found. Good $\stackrel{D}{2}$ growth occurred on unenriched nutrient agar and in $\stackrel{\infty}{+}$ peptone water media. The failure to grow on CLED 
agar therefore suggested a requirement for salt. This was readily proved by depositing a small amount of sodium chloride crystals on the inoculated area of a CLED plate; profuse growth occurred in the area surrounding the deposit. Using varying concentrations of $\mathrm{NaCl}$ in nutrient agar, it was shown that although there was no growth without added salt, good growth occurred on $1.0 \%$ and on $5.0 \%$ with moderate growth on $10.0 \%$.

Although adequate growth appeared at $37^{\circ} \mathrm{C}$ it was found that the optimum temperature was about $30^{\circ} \mathrm{C}$ with good growth also at $20^{\circ} \mathrm{C}$ but no growth at $42^{\circ} \mathrm{C}$. There was a tendency to swarming on nutrient agar plates at $20^{\circ} \mathrm{C}$. MacConkey agar gave rather poor growth of non-lactose fermenting colonies. A selective medium for vibrios, TCBS (Oxoid), gave good growth of large, smooth yellow colonies. Cultures were non-luminous even on high salt-containing agar.

The following biochemical tests were positive: oxidase, catalase, reduction of nitrate to nitrite, gelatinase, lysine decarboxylase, and VP test. The following were negative: urease, phenylalanine deaminase, malonate and citrate utilization, arginine dehydrolase, and the MR test. Indole was produced but in only small amounts.

Acid but no gas was produced from carbohydrates by fermentation. The following showed acid production, usually in one or two days: glucose, fructose, mannitol, mannose, glycerol, galactose, maltose, trehalose, and sucrose. No acid was formed in 14 days from the following: arabinose, sorbitol, dulcitol, adonitol, inositol, xylose, lactose, raffinose, and rhamnose.

Three per cent sodium chloride was incorporated in glucose-phosphate peptone water for VP and MR tests. Otherwise routine media without increased salt were found to be satisfactory.

All cultures were sensitive to the vibriostatic agent 0/129 (2,4-diamino-6,7-di-iso-propylpteridine phosphate). Disc tests showed sensitivity to tetracycline, streptomycin, gentamicin, cotrimoxazole, and colistin with resistance to penicillin, ampicillin, and cephaloridine.

From the foregoing results it appeared that these bacteria were marine vibrios requiring for growth the presence of sodium chloride, or perhaps more specifically the sodium ion as demonstrated with other halophiles. Swarming, growth in $10 \% \mathrm{NaCl}$, acid from sucrose but not arabinose, and the results of the VP and MR tests indicated $V$. alginolyticus rather than $V$. parahaemolyticus, while swarming, lack of acid from sorbitol, and the results with lysine and arginine indicated $V$. alginolyticus rather than $V$. anguillarium. Results with the other tests supported this identification which was confirmed through the kindness of Dr G. I. Barrow of the Public Health Laboratory, Truro.

\section{Discussion}

The marine vibrios $V$. parahaemolyticus, $V$. alginolyticus, and $V$. anguillarium are normally found in marine animals and in inshore sea-water. In the United States Twedt et al (1969) examined 14 isolates of marine vibrios obtained from localized tissue infections in 'swimmers and others in recreational contact with the marine environment' and compared them with Japanese isolates of typical $V$. parahaemolyticus. These authors concluded that the tissue isolates were probably $V$. parahaemolyticus but a study of their results suggests that the group was not homogeneous. This was confirmed by Zen-Yoji et al (1973), who re-examined the same strains and found that six of them were identifiable as $V$. parahaemolyticus, six as $V$. alginolyticus, and two were unidentifiable vibrios. Thus it would seem that either of these two species can be associated with local tissue infection in exposed individuals. In the present report all three isolates undoubtedly had the characteristics of $V$. alginolyticus. It is worth noting that the 'comma' shape, commonly associated with vibrios, is not necessarily a prominent feature in the morphology of these species.

Although the evidence is not conclusive, it seems likely that the association of marine vibrios with superficial septic lesions may be of pathological significance. Further investigations are required to confirm this and also to define the prevalence of the condition, its possible course, and optimum treatment. It would perhaps be advisable for general practitioners, hospitals, and laboratories serving coastal areas to be aware of the possibility of such an infection and to be especially vigilant during the holiday season.

\section{References}

Cowan, S. T. (1974). Cowan and Steel's Manual for the Identification of Medical Bacteria, 2nd edition. Cambridge University Press, London.

Mackey, J. P. and Sandys, G. H. (1966). Diagnosis of urinary infections. (Letter). Brit. med. J., 1, 1173.

Twedt, R. M., Spaulding, P. L., and Hall, H. E. (1969). Morphological, cultural, biochemical, and serological comparison of Japanese strains of Vibrio parahemolyticus with related cultures isolated in the United States. J. Bact., 98, 511-518.

Zen-Yoji, H., Leclair, R. A., Ohta, K., and Montague, T. S. (1973). Comparison of Vibrio parahaemolyticus cultures isolated in the United States with those isolated in Japan. J. infect. Dis., 127, 237-241. 